\section{Laser scanning in vivo confocal microscopy and quantitative aesthesiometry reveal decreased corneal innervation and sensation in keratoconus}

DV Patel, JYF Ku, R Johnson and CNJ McGhee
Department of

Ophthalmology, Faculty of Medical and Health Sciences, University of Auckland, Auckland, New Zealand

Correspondence:

CNJ McGhee,

Department of

Ophthalmology,

Faculty of Medical and

Health Sciences,

University of Auckland,

Private Bag 92019,

Auckland 1 ,

New Zealand

Tel: +6493737599

ext. 86712

Fax: + 6493677173

E-mail: c.mcghee@

auckland.ac.nz

Received: 1 October 2007 Accepted in revised form: 4 February 2008

Published online: 14 March 2008

\begin{abstract}
Purpose The purpose of this study was to quantitatively analyse laser scanning in vivo confocal microscopy images of the corneal epithelium and sub-basal nerve plexus in patients with keratoconus and to correlate these microstructural observations with corneal sensitivity.

Methods A total of 31 eyes of 31 normal human subjects, and 27 eyes of 27 subjects with an established diagnosis of keratoconus were recruited. Twelve subjects with keratoconus had never worn contact lenses (K-NCL). Fifteen subjects with keratoconus wore contact lenses routinely (K-CL). All eyes were examined using slit-lamp biomicroscopy, Orbscan topography, non-contact corneal aesthesiometry, and laser scanning in vivo confocal microscopy.

Results Central corneal sensation was significantly lower in K-CL compared to normal $(P=0.028)$. However, there was no significant difference in corneal sensation between the normal and K-NCL groups $(P=0.059)$. Both sub-basal nerve density $(P<0.001)$ and basal epithelial density $(P<0.001)$ were significantly lower than normal in all keratoconic subjects. Central corneal sensation was only significantly correlated with sub-basal nerve density $(P=0.001)$ and was not significantly correlated with any of the basal epithelial parameters. Sub-basal nerve density showed significant positive correlation with basal epithelial density $(P<0.001)$.
\end{abstract}

Conclusion This quantitative study reveals decreased corneal innervation, sensation, and basal epithelial density in keratoconus. The results of this study provide strong evidence that both the sub-basal nerves and the basal epithelium may be involved in the pathogenesis of keratoconus, although it is uncertain whether these are primary or secondary changes.

Eye (2009) 23, 586-592; doi:10.1038/eye.2008.52; published online 14 March 2008

Keywords: cornea; keratoconus; in vivo confocal microscopy; epithelium; nerves; sensation

\section{Introduction}

Keratoconus is a non-inflammatory disorder in which the cornea assumes a conical shape due to thinning and protrusion. ${ }^{1}$ Classically, onset is at puberty, with progression until the third or fourth decade of life, when it usually arrests. ${ }^{2}$ Clinically, this corneal ectasia leads to myopia and irregular astigmatism, and in severe cases, ruptures in Descemet's membrane may occur, resulting in corneal oedema and scarring. ${ }^{1}$ The pathophysiology of keratoconus has yet to be fully resolved though there appears to be an underlying genetic predisposition. ${ }^{3}$

There is no animal model for keratoconus, so the majority of research investigating the pathogenesis of keratoconus has involved the examination of corneal buttons excised during penetrating keratoplasty. These studies have 
demonstrated histopathological abnormalities in every layer of the keratoconic cornea. ${ }^{4}$ One major limitation of such studies is that due to the tissue source typically they only sample the very severe end of the disease spectrum. In vivo confocal microscopy overcomes this problem by enabling examination of the living human cornea at the cellular level. The non-invasive nature of this technique means that multiple examinations may be performed on the same tissue over time, and the induction of artefacts observed with ex vivo methods of examination are avoided.

The purpose of this study was to quantitatively analyse laser scanning in vivo confocal microscopy images of the corneal epithelium and sub-basal nerve plexus in patients with keratoconus and to correlate these microstructural observations with corneal sensitivity and severity of disease.

\section{Methods}

\section{Subjects}

Following informed consent, 31 normal healthy human subjects, and 27 subjects with an established diagnosis of keratoconus were recruited. One eye of each subject was included in the study and exclusion criteria were history of ocular trauma or surgery, ocular disease (other than keratoconus), and systemic diseases that may affect the cornea (due to the relative infrequency of rigid gas permeable (RGP) contact lens wear in this age group, a control group of normal subjects who wore RGP contact lenses could not be recruited). Those subjects with keratoconus who routinely wore contact lenses were requested to temporarily cease wearing contact lens for $12 \mathrm{~h}$ prior to examination. For keratoconic subjects in whom both eyes were eligible for the study, one eye was selected at random.

Informed, written consent was obtained from all subjects after explanation of the nature and possible consequences of the study. The study protocol was approved by the Auckland Ethics Committee.

\section{Examinations}

Slit-lamp biomicroscopy was performed on all eyes and each subject exhibited one or more of the following clinical signs: central corneal stromal thinning, Fleisher's ring, Vogt's striae, or Munson's sign. No eyes had evidence of corneal hydrops or scarring.

Corneal sensitivity was measured by non-contact pneumatic corneal aesthesiometry ${ }^{5}$ using a $10 \mathrm{~mm}$ working distance and $0.9 \mathrm{~s}$ stimulus duration. All subjects were asked to fixate on a distance target to enable measurements on the central cornea.
In all cases Orbscan II-combined Placido/slit-scanning elevation topography (Bausch and Lomb Surgical, Rochester, NY, USA) was performed to confirm the clinical diagnosis and to further classify the severity of keratoconus. The modified Rabinowitz-McDonnell test was used to confirm the diagnosis of keratoconus, ${ }^{6}$ and the severity of keratoconus was classified according to the steepest simulated keratometry reading on the keratometric map (mild $<45 \mathrm{D}$, moderate 45-52 D, severe $>52 \mathrm{D})$.

Laser scanning in vivo confocal microscopy was subsequently performed on all subjects using the Heidelberg Retina Tomograph II Rostock Corneal Module (RCM; Heidelberg Engineering GmBH, Germany). This microscope utilises a $670 \mathrm{~nm}$ red wavelength diode laser source - a class 1 laser system that by definition, does not pose any ocular safety hazard. However, to guarantee the safety of the operator and subjects, the manufacturers have imposed a limit on the maximum period of exposure for patient and operator of $3000 \mathrm{~s}(50 \mathrm{~min})$ in any single examination period. A $\times 60$ objective water immersion lens with a numerical aperture of 0.9 (Olympus, Japan) and a working distance, relative to the applanating cap, of 0.0-3.0 $\mathrm{mm}$ was used. The dimensions of each image produced using this lens are $400 \times 400 \mu \mathrm{m}$, and the manufacturers quote the transverse resolution and optical section thickness as 2 and $4 \mu \mathrm{m}$ respectively. The RCM uses an entirely digital image capture system.

All eyes were anaesthetised using a drop of $0.4 \%$ benoxinate hydrochloride (Chauvin Pharmaceuticals). Viscotears (Carbomer 980, 0.2\%; Novartis, Australia) was used as a coupling agent between the applanating lens cap and the cornea. During the examination, all subjects were asked to fixate on a distance target aligned to enable examination of the central cornea. The full thickness of the central cornea was scanned using the device's 'section mode'. The section mode enables instantaneous imaging of a single area of the cornea at a desired depth. The overall examination took approximately $10 \mathrm{~min}$ to perform for each subject and none of the subjects experienced any visual symptoms or corneal complications as a result of examination.

\section{Image analysis}

An experienced observer (DVP) selected two frames per location that contained the clearest images of the basal epithelium and the maximum number of nerves at the level of the sub-basal plexus. All frames were subsequently randomised and encoded by an independent observer (JYFK). Measurements were then performed (DVP) using a calliper tool (analySIS 3.1, Soft Imaging System, Münster, Germany). 
For all epithelial images, a standard frame size of $100 \times 100 \mu \mathrm{m}$ was selected. For all sub-basal plexus images, a standard frame size of $300 \times 300 \mu \mathrm{m}$ was selected.

The mean basal epithelial diameter was determined by measuring the longest diameter and the diameter of its perpendicular bisector for 10 cells. The area of 10 basal epithelial cells and the density of cells within the frame were also measured. The sub-basal nerve density was assessed by measuring the total length of nerves per frame.

\section{Statistical analysis}

SPSS version 12 for windows (Chicago, IL, USA) was used for statistical analysis.

The Kruskal-Wallis test was used to determine any significant differences between multiple groups. The Mann-Whitney $U$-test was used to compare the means of variables between pairs of groups. $P$-values of $<0.05$ were considered significant for all statistical tests.

\section{Results}

The age-matched control group consisted of 31 normal subjects with healthy corneas, consisting 13 men and 18 women with a mean age of $35 \pm 12$ years. The keratoconus study group, consisted of 27 subjects with 14 men and 13 women $(\mathrm{M}: \mathrm{F}=14: 13)$ with a mean age of $37 \pm 10$ years. Twelve subjects $(\mathrm{M}: \mathrm{F}=6: 6,37 \pm 9$ years $)$ with keratoconus had never worn contact lenses (K-NCL). Fifteen subjects (M:F=8:7, 37 \pm 11 years) with keratoconus wore contact lenses routinely (K-CL).

Fourteen of these subjects wore RGP contact lenses and one wore a soft contact lens. The mean duration of contact lens wear in the K-CL group was $10.3 \pm 9.4$ years, for a mean of $12.3 \pm 4.6 \mathrm{~h}$ per day.

Of the 27 corneas with keratoconus, on the basis of Orbscan II topography, 4 were classified as mild, 10 as moderate, and 13 as severe cones.
When keratoconic corneas were imaged by laser scanning in vivo confocal microscopy, qualitatively the sub-basal nerves appeared to be more tortuous (Figure 1) and the basal epithelial cells appeared to show greater variability in shape and size compared to the normal cornea (Figure 2).

When analysed according to group using the KruskalWallis test, all parameters demonstrated significant differences between the groups (Table 1). Post hoc MannWhitney $U$-tests on pairs of groups revealed that central corneal sensation was significantly lower in K-CL compared to normal group $(P=0.028)$. However, there was no significant difference in corneal sensation between the normal and K-NCL groups $(P=0.059)$.

The mean sub-basal nerve density was significantly lower in both K-NCL $(P<0.001)$ and K-CL $(P<0.001)$ groups compared to normal group.

The mean basal epithelial densities of K-NCL and K-CL were significantly lower than normal $(P<0.001$ for each group). Additionally, the mean basal epithelial diameters and areas were correspondingly greater and this difference was significant $(P<0.001)$ between each group.

When analysed according to severity, using the Kruskal-Wallis test, all parameters demonstrated significant differences between the severity groups (Table 2). Post hoc Mann-Whitney $U$-tests on pairs of
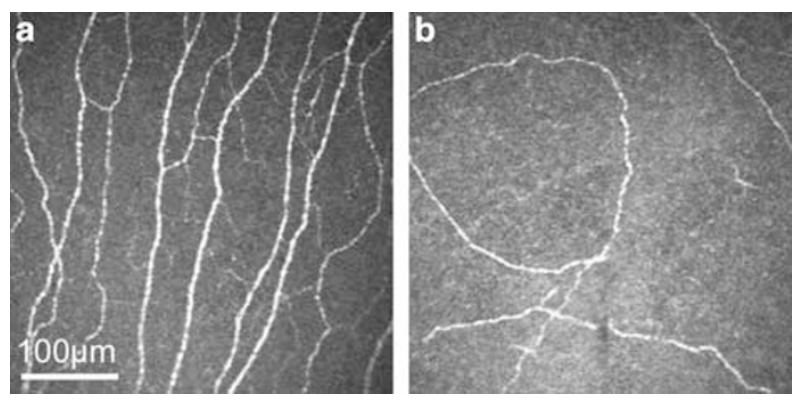

Figure 1 In vivo confocal images of the sub-basal nerve plexus in the normal cornea (a), and in keratoconus associated with rigid gas permeable contact lens wear $(b)$.
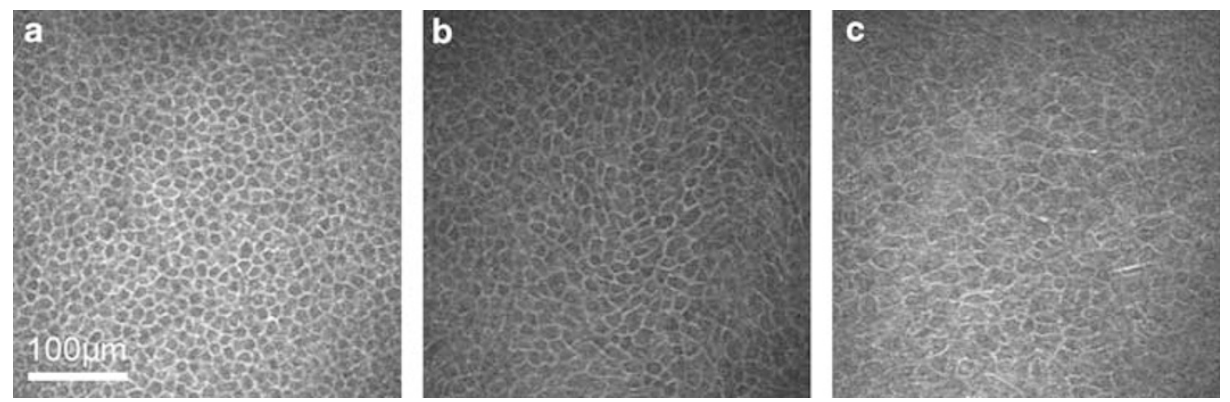

Figure 2 In vivo confocal microscopy of the basal epithelium in normal cornea (a), in keratoconus with no history of contact lens wear (b), and in keratoconus associated with rigid gas permeable contact lens wear (c). 
Table 1 Comparison of corneal sensitivity threshold, sub-basal nerve density, and basal epithelial parameters (diameter, area, and density) for each of three groups (normal, K-NCL, and K-CL)

\begin{tabular}{|c|c|c|c|c|c|c|}
\hline & $\begin{array}{c}\text { Eyes } \\
\text { (n) }\end{array}$ & $\begin{array}{l}\text { Mean central corneal } \\
\text { sensitivity threshold } \\
\pm S D \text { (mBar) }\end{array}$ & $\begin{array}{c}\text { Mean sub-basal } \\
\text { nerve density } \pm S D \\
\left(\mu \mathrm{m} / \mathrm{mm}^{2}\right)\end{array}$ & $\begin{array}{l}\text { Mean basal epithelial } \\
\text { diameter } \pm S D(\mu m)\end{array}$ & $\begin{array}{l}\text { Mean basal epithelial } \\
\text { area } \pm S D\left(\mu m^{2}\right)\end{array}$ & $\begin{array}{c}\text { Mean basal epithelial } \\
\text { density } \pm S D \\
\left(\text { cells } / \mathrm{mm}^{2}\right)\end{array}$ \\
\hline Normal & 31 & $0.35 \pm 0.11$ & $25929 \pm 6968$ & $13.1 \pm 0.8$ & $154.0 \pm 16.0$ & $5823 \pm 602$ \\
\hline K-NCL & 12 & $0.40 \pm 0.12$ & $16082 \pm 8188$ & $14.3 \pm 1.7$ & $180.2 \pm 34.2$ & $5188 \pm 765$ \\
\hline $\mathrm{K}-\mathrm{CL}$ & 15 & $0.48 \pm 0.25$ & $12140 \pm 6739$ & $15.8 \pm 2.0$ & $221.9 \pm 66.3$ & $4428 \pm 1056$ \\
\hline Kruskal-Wallis & & $P=0.038$ & $P<0.001$ & $P<0.001$ & $P<0.001$ & $P<0.001$ \\
\hline
\end{tabular}

Abbreviations: K-CL, contact lens wearing keratoconics; K-NCL, non-contact lens wearing keratoconics.

When analysed according to group, using the Kruskal-Wallis test, all parameters demonstrated significant differences between the groups.

Table 2 Comparison of corneal sensitivity threshold, sub-basal nerve density, and basal epithelial parameters (diameter, area, and density) for different severities of keratoconus

\begin{tabular}{lcccccc}
\hline & $\begin{array}{c}\text { Eyes } \\
(\mathrm{n})\end{array}$ & $\begin{array}{c}\text { Mean central corneal } \\
\text { sensitivity threshold } \\
\pm S D(\text { mBar })\end{array}$ & $\begin{array}{c}\text { Mean sub-basal } \\
\text { nerve density } \pm S D \\
\left(\mu \mathrm{m} / \mathrm{mm}^{2}\right)\end{array}$ & $\begin{array}{c}\text { Mean basal } \\
\text { epithelial diameter } \\
\pm S D(\mu \mathrm{m})\end{array}$ & $\begin{array}{c}\text { Mean basal } \\
\text { epithelial area } \\
\pm S D\left(\mu m^{2}\right)\end{array}$ & $\begin{array}{c}\text { Mean basal epithelial } \\
\text { density } \pm S D \\
\left(\text { cells }^{2} \mathrm{~mm}^{2}\right)\end{array}$ \\
\hline Normal & 31 & $0.35 \pm 0.11$ & $25929 \pm 6,968$ & $13.1 \pm 0.8$ & $154.0 \pm 16.0$ & $5823 \pm 602$ \\
Mild & 4 & $0.44 \pm 0.16$ & $14204 \pm 8057$ & $13.9 \pm 1.0$ & $170.0 \pm 23.5$ & $5225 \pm 654$ \\
Moderate & 10 & $0.39 \pm 0.21$ & $17566 \pm 7620$ & $15.1 \pm 2.2$ & $202.6 \pm 61.8$ & $4945 \pm 1080$ \\
Severe & 13 & $0.49 \pm 0.21$ & $10971 \pm 6375$ & $15.6 \pm 1.9$ & $213.9 \pm 59.4$ & $4488 \pm 975$ \\
Kruskal-Wallis & & $P=0.01$ & $P<0.001$ & $P<0.001$ & $P<0.001$ & $P<0.001$ \\
\hline
\end{tabular}

When analysed according to severity, using the Kruskal-Wallis test, all parameters demonstrated statistically significant differences between the severity groups.

Table 3 Spearman's $\rho$ correlations for corneal sensitivity threshold, sub-basal nerve density, and basal epithelial parameters (diameter, area, and density)

\begin{tabular}{lcccc}
\hline & $\begin{array}{c}\text { Central } \\
\text { corneal } \\
\text { sensation }\end{array}$ & $\begin{array}{c}\text { Sub-basal } \\
\text { nerve } \\
\text { density }\end{array}$ & $\begin{array}{c}\text { Basal } \\
\text { epithelial } \\
\text { diameter }\end{array}$ & $\begin{array}{c}\text { Basal } \\
\text { epithelial } \\
\text { area }\end{array}$ \\
\hline $\begin{array}{l}\text { Sub-basal nerve density } \\
\text { Correlation } \\
\text { coefficient }\end{array}$ & -0.31 & & & \\
$P$ & $0.001^{*}$ & & & \\
$\begin{array}{l}\text { Basal epithelial diameter } \\
\text { Correlation } \\
\text { coefficient }\end{array}$ & 0.13 & -0.43 & & \\
$P$ & 0.167 & $<0.001^{*}$ & & \\
$\begin{array}{l}\text { Basal epithelial area } \\
\text { Correlation } \\
\text { coefficient }\end{array}$ & 0.02 & -0.36 & 0.89 & \\
$P$ & 0.837 & $<0.001^{*}$ & $<0.001^{*}$ & \\
$\begin{array}{l}\text { Basal epithelial density } \\
\text { Correlation } \\
\text { coefficient }\end{array}$ & -0.10 & 0.48 & -0.87 & -0.84 \\
$P$ & 0.283 & $<0.001^{*}$ & $<0.001^{*}$ & $<0.001^{*}$ \\
\hline
\end{tabular}

${ }^{*}$ Correlation is significant at the 0.01 level (two tailed).

groups revealed that with the exception of corneal sensation in the normal verses moderate keratoconus groups, all parameters were significantly different between the normal group and each keratoconus severity group.

There were no significant differences in any of the parameters when the mild and moderate keratoconus groups were compared. The mild and severe keratoconus groups demonstrated significant differences in sub-basal nerve density, and epithelial diameter and area. The moderate and severe groups were only significantly different in sub-basal nerve density.

Spearman's correlation (Table 3) revealed that central corneal sensation was only significantly correlated with sub-basal nerve density $(P=0.001)$ and was not significantly correlated with any of the basal epithelial parameters. Sub-basal nerve density showed significant positive correlation with basal epithelial density $(P<0.001)$ and corresponding negative correlations with basal epithelial diameter and area $(P<0.001$ each).

\section{Discussion}

We believe on the basis of literature review that the reported study is the first to investigate corneal sensation and severity of keratoconus using the quantitative noncontact pneumatic corneal aesthesiometer. There was no significant difference in corneal sensitivity between the K-NCL group and the normal group, while contact lens wear in keratoconic corneas was associated with significantly decreased corneal sensation compared to normal. Additionally, there was no clear association 
between severity and corneal sensation. This study would be enhanced by the inclusion of a control group of RGP contact les wearers by enabling differentiation of the effects of contact lens wear and those of keratoconus. However, due to the relative infrequency of RGP contact lens wear in normal subjects in this age group, we were unable to recruit such a group.

Previous studies, using the Cochet-Bonnet aesthesiometer, have reported central corneal sensation to be significantly lower than normal in non-contact lens wearing keratoconic eyes, and even lower in keratoconic eyes wearing contact lenses. ${ }^{7,8}$ A significant correlation between central corneal sensation and severity of keratoconus has also been reported, although only in non-contact lens wearers. ${ }^{7}$ The lack of association between these parameters in the current study may be attributed to the small sample size and the differences in severity group sizes. In a more recent study by Simo Mannion et al, ${ }^{9}$ subjects with keratoconus who wore contact lenses had significantly lower corneal sensation compared to normal controls who wore contact lenses. The differences between the results of previous studies and the current one may be attributed to the different methods of assessing corneal sensitivity. The CochetBonnet aesthesiometer measures corneal touch threshold and has a limited range of stimuli, often at suprathreshold levels. The non-contact pneumatic aesthesiometer has a larger range of stimulus intensities and measures the corneal nerve threshold to a composite stimulus consisting of air pressure, tear evaporation, and disruption. ${ }^{5}$

Previously, the majority of in vivo data regarding alterations in the sub-basal nerve plexus have been limited to qualitative studies, with observations such as 'sub-basal nerve fibres running in and out of the plane of the field of view in the central cornea' ${ }^{10}$ In the current study, the sub-basal nerves qualitatively appeared more tortuous in keratoconic corneas compared to controls. This observation concurs with the results of Simo Mannion $e t a l^{9}$ who noted a greater range of orientations of sub-basal nerve fibres in keratoconic eyes compared with controls. A recent study has demonstrated grossly abnormal sub-basal nerve architecture in keratoconus by producing two-dimensional reconstruction maps using in vivo confocal microscopy. ${ }^{11}$ At the apex of the cone, a tortuous network of nerve fibre bundles was noted, many of which formed closed loops. At the topographic base of the cone, nerve fibre bundles appeared to follow the contour of the base, with many of the bundles running concentrically in this region.

The current study demonstrated that the sub-basal nerve density decreased with severity of disease and was significantly lower than normal corneas in the K-NCL group, and even lower in the K-CL group. We have recently reported that keratocyte density is also significantly lower in keratoconus with the decline in density correlating with the severity of the keratoconus. ${ }^{12}$ However, although a previous quantitative analysis of corneal nerves in moderate keratoconus demonstrated significantly reduced sub-basal nerve density in contact lens wearing keratoconic subjects compared with contact lens wearing controls, unlike the current study, there was no significant difference in sub-basal nerve density in the non-contact lens wearing subjects in the keratoconus and control groups. ${ }^{9}$ This latter difference may be due to the smaller number of subjects in the previous study.

The accurate measurement of sub-basal nerve diameter might provide a very useful additional parameter. However, for measurements of the diameter of thin highly reflective structures (such as sub-basal nerves) to be comparable, all images need to be acquired using a fixed illumination intensity since illumination intensity affects the apparent thickness of corneal nerves. In the current study, the automatic brightness setting was used such that the proprietary software automatically adjusted the brightness of the live image to maximise image quality. We considered maximum image quality to be the greatest priority for measuring the key parameters included in the study and therefore did not measure sub-basal nerve diameter.

Overall, these observations suggest a role for corneal nerves in the pathophysiology of keratoconus. The results of ex vivo studies support this theory. A transmission electron microscopy study of keratoconic corneal discs removed at penetrating keratoplasty noted that nerve fibres within the corneal epithelium showed signs of moderate degeneration, with break up of the nerve fibre membrane and liquefaction of neurofibrils. ${ }^{13}$ Brookes $e t ~ a l^{14}$ provided evidence that the destructive process in keratoconus involved the nerves, or their associated Schwann cells, which express proteolytic enzymes (cathepsin B and G) more extensively in keratoconus compared to normal corneas.

In vivo studies of the corneal epithelium in keratoconus, using slit scanning in vivo confocal microscopy, have revealed variable basal cell appearances. Somodi et $a l^{15}$ noted that the basal cell layer exhibited highly reflective structures and fold-like changes in the apical region, while a regular basal cell pattern was observed in the corneal periphery. Hollingsworth et a $l^{10}$ reported that $12 \%$ of eyes exhibited a highly irregular basal cell layer appearance, with visible nuclei and the majority of eyes in this group were classified as having severe keratoconus. Quantitative analysis has revealed a significantly greater mean basal epithelial diameter in keratoconic patients compared to that of normal subjects, though there were no data regarding cell density. ${ }^{16}$ Neither of these studies made a 
distinction between contact lens wearers and nonwearers during data analysis. Uçakhan ${ }^{17}$ recently reported contradictory results, with keratoconics demonstrating mean basal epithelial cell densities approximately double that of normal subjects. This discrepancy was attributed to differences in the study groups (subjects in the latter study had never worn contact lenses).

The current study extends previous observations demonstrating that basal epithelial density was significantly lower in the K-NCL group and even lower in the K-CL compared to normal group. However, although epithelial density appeared to decline with increased severity of keratoconus, these differences were not significant. These results suggest that the basal epithelium may be involved in the pathophysiology of keratoconus, and is further affected by contact lens wear. This hypothesis is supported by histological and electron microscopy studies of corneal buttons removed at penetrating keratoplasty. Teng ${ }^{13}$ identified degenerative changes in the basal epithelium, with dead cells in this layer mostly present in the central cornea. This led to the suggestion that these cells may be the site of the primary lesion in keratoconus.

The reported study quantitatively confirms a significant correlation between sub-basal nerve density and corneal sensation. Previous studies have attempted to determine the relationship between sub-basal nerve structure and function with varying results. Some have noted a significant correlation between corneal sensitivity and sub-basal nerve morphology, ${ }^{18}$ or the number of long nerve fibre bundles visible per frame, ${ }^{19}$ while others have found no significant correlation between these variables. ${ }^{20}$ All of these studies differed from the current study in that corneal sensitivity was assessed using the Cochet-Bonnet aesthesiometer. Additionally, the method of analysis of in vivo confocal images differed from that of the current study.

The confirmation of a positive correlation between subbasal nerve density and basal epithelial density reported here concurs with our knowledge of the close relationship between corneal innervation and the maintenance of epithelial proliferation and wound healing obtained from clinical ${ }^{21}$ and experimental ${ }^{22}$ observations.

This is the first study to quantitatively analyse the central corneal sensation, sub-basal nerve density, and epithelial density in keratoconus. The results of this study provide strong evidence that both the sub-basal nerves and the basal epithelium may be involved in the pathogenesis of keratoconus, although it is uncertain whether these are primary or secondary changes. Future studies may be directed at examining subjects with keratoconus over time to observe whether changes in sub-basal nerve architecture precede, or follow progression of the disease.

\section{Acknowledgements}

This study was supported in part by an unrestricted award from The Maurice and Phyllis Paykel Trust.

The authors have no proprietary interest in any of the devices mentioned in this article.

\section{References}

1 Krachmer JH, Feder RS, Belin MW. Keratoconus and related noninflammatory corneal thinning disorders. Surv Ophthalmol 1984; 28(4): 293-322.

2 Rabinowitz YS. Keratoconus. Surv Ophthalmol 1998; 42(4): 297-319.

3 Edwards M, McGhee CN, Dean S. The genetics of keratoconus. Clin Experiment Ophthalmol 2001; 29(6): 345-351.

4 Sherwin T, Brookes NH. Morphological changes in keratoconus: pathology or pathogenesis. Clin Experimen Ophthalmol 2004; 32(2): 211-217.

5 Murphy PJ, Patel S, Marshall J. A new non-contact corneal aesthesiometer (NCCA). Ophthalmic Physiol Opt 1996; 16(2): 101-107.

6 Maeda N, Klyce SD, Smolek MK. Comparison of methods for detecting keratoconus using videokeratography. Arch Ophthalmol 1995; 113(7): 870-874.

7 Millodot M, Owens H. Sensitivity and fragility in keratoconus. Acta Ophthalmologica 1983; 61(5): 908-917.

8 Zabala M, Archila EA. Corneal sensitivity and topogometry in keratoconus. CLAO J 1988; 14(4): 210-212.

9 Simo Mannion L, Tromans C, O'Donnell C. An evaluation of corneal nerve morphology and function in moderate keratoconus. Contact Lens Anterior Eye 2005; 28(4): 185-192.

10 Hollingsworth JG, Bonshek RE, Efron N. Correlation of the appearance of the keratoconic cornea in vivo by confocal microscopy and in vitro by light microscopy. Cornea 2005; 24(4): 397-405.

11 Patel DV, McGhee CN. Mapping the corneal sub-basal nerve plexus in keratoconus by in vivo laser scanning confocal microscopy. Invest Ophthalmol Vis Sci 2006; 47(4): 1348-1351.

$12 \mathrm{Ku}$ JY, Niederer RL, Patel DV, Sherwin T, McGhee CNJ. Laser scanning in vivo confocal analysis of keratocyte density in keratoconus. Ophthalmology 2007, e-pub ahead of print 5 September 2007; PMID: 17825419.

13 Teng CC. Electron microscopy study of the pathology of keratoconus. Am J Ophthalmol 1963; 55: 18-47.

14 Brookes NH, Loh IP, Clover GM, Poole CA, Sherwin T. Involvement of corneal nerves in the progression of keratoconus. Exp Eye Res 2003; 77(4): 515-524.

15 Somodi S, Hahnel C, Slowik C, Richter A, Weiss DG, Guthoff R. Confocal in vivo microscopy and confocal laserscanning fluorescence microscopy in keratoconus. German J Ophthalmol 1996; 5(6): 518-525.

16 Hollingsworth JG, Efron N, Tullo AB. In vivo corneal confocal microscopy in keratoconus. Ophthalmic Physiol Opt 2005; 25(3): 254-260. 
17 Uçakhan OO, Kanpolat A, Ylmaz N, Ozkan M. In vivo confocal microscopy findings in keratoconus. Eye Contact Lens 2006; 32(4): 183-191.

18 Linna TU, Vesaluoma MH, Perez-Santonja JJ, Petroll WM, Alió JL, Tervo TM. Effect of myopic LASIK on corneal sensitivity and morphology of subbasal nerves. Invest Ophthalmol Vis Sci 2000; 41(2): 393-397.

19 Rosenberg ME, Tervo TM, Immonen IJ, Müller LJ, Grönhagen-Riska C, Vesaluoma MH. Corneal structure and sensitivity in type 1 diabetes mellitus. Invest Ophthalmol Vis Sci 2000; 41(10): 2915-2921.
20 Patel SV, McLaren JW, Hodge DO, Bourne WM. Confocal microscopy in vivo in corneas of long-term contact lens wearers. Invest Ophthalmol Vis Sci 2002; 43(4): 995-1003.

21 Davies MS. Corneal anaesthesia after alcohol injection of the trigeminal sensory root. Examination of 100 anaesthetic corneae. Br J Ophthalmol 1970; 54(9): 577-586.

22 Beuerman RW, Schimmelpfennig B. Sensory denervation of the rabbit cornea affects epithelial properties. Exp Neurol 1980; 69(1): 196-201. 